\title{
A COMPARATIVE STUDY OF MICRORHEOLOGY OF NANOCELLULOSE PRODUCED FROM CORN STOVER USING DIFFUSING WAVE SPECTROSCOPY (DWS) AND MECHANICAL RHEOMETRY
}

\author{
JINGYUAN XU, VEERA M. BODDU, SEAN X. LIU* and WEN-CHING LIU** \\ *National Center for Agricultural Utilization Research, Agricultural Research Service, United States \\ Department of Agriculture, 1815 North University Street, Peoria, Illinois 61604, USA \\ ${ }^{* *}$ University of Illinois, College of Medicine at Peoria, Peoria, Illinois 61605, USA \\ $₫$ Corresponding author: Jingyuan Xu, james.xu@usda.gov
}

Received July, 16, 2019

\begin{abstract}
Agricultural waste corn stover has very little value, but can be a good renewable, biodegradable, and inexpensive source to produce value-added products, such as cellulose and nanocellulose. The properties and functions of cellulose and nanocellulose depend on the cellulose source and preparation process. The microheterogeneity and microrheological properties of corn stover nanocellulose (CSNC) solutions were investigated by a novel technique named diffusing wave spectroscopy (DWS); and both DWS and mechanical rheometry measurements for the prepared CSNC were compared and analyzed. We expected that the DWS study of the CSNC could reveal some new insight into the CSNC properties. It is also expected that the microrheological behavior of CSNC measured by DWS should be matched with the data measured by conventional mechanical rheometry. By comparing the meansquare displacement (MSD) of the microbeads embedded in five concentrations of CSNC solutions measured by DWS, we found that the solutions exhibited slight heterogeneity behavior at the lower concentration of $0.25 \%$, while the material displayed a definite degree of heterogeneity at higher concentrations. DWS microrheology measurements were in excellent agreement with the conventional mechanical rheological studies for CSNC. The magnitude of the high-frequency viscoelastic moduli $\left(\left|G^{*}(\omega)\right|\right)$ for the CSNC was proportional to the $\omega^{0.75}$, which entails semi-flexible polymer behavior. The identified properties of the CSNC will provide useful information for utilizing this kind of nanocellulose in various applications.
\end{abstract}

Keywords: corn stover, diffusing wave spectroscopy (DWS), microrheology, nanocellulose

\section{INTRODUCTION}

Cellulose is the most abundant of the natural polymers on earth. It can be obtained from many sources, such as crop plants, wood, cotton, and agricultural wastes. ${ }^{1}$ The composition, structure, and function of cellulose highly rely on its sources. $^{2,3}$ In recent decades, scientists have focused on nanocellulose (NC) with a focus on its application in nanoscience and nanotechnology. ${ }^{1}$ Nanocellulose possesses unique and promising properties, such as high mechanical strength and low density. ${ }^{3}$ Many agricultural wastes, such as corn stover, sorghum stalks, and wheat straw are inexpensive, abundant sources of cellulose. These residues are generally categorized as wastes because they have very little economic value. Agricultural waste materials are excellent resources of value-added materials, such as cellulose and NC, because they are biodegradable, inexpensive, renewable, and readily available.

Diffusing wave spectroscopy (DWS), which is a novel technology, can be used to investigate the microheterogeneity and microrheological properties of polymer solutions and gels. ${ }^{4,5}$ Employing the DWS, the material's viscoelastic moduli over a wide frequency range, at a certain temperature, can be tested in a single measurement, without interfering with the sample. ${ }^{4-6}$ It monitors the thermal-driven movements of microbeads that are embedded in a polymer solution or gel. The mean square displacements (MSD) of the probed microspheres can be monitored with a nano-second resolution 
by DWS. From MSD and the diffusion coefficients calculated from MSD, one can get an insight into the polymer solution's microrheological properties. ${ }^{5,6}$ The viscoelastic moduli for the samples can be extracted from the measured MSD using the generalized StokesEinstein equation. ${ }^{7}$ The DWS can measure the rheological behaviors of the sample as high as $10^{6}$ $\mathrm{rad} / \mathrm{s}$, which are nearly three orders of magnitude greater than probed by mechanical rheometers. In this way, an understanding of the type of local macromolecular movement that produces stress relaxation at very short times, as well as a new insight into the mechanism of early dynamics of stress relaxation in polymer networks can be obtained. The DWS technology has been used to study some biopolymers, such as actin, ${ }^{6} \beta$ glucan, ${ }^{5}$ and wheat protein. ${ }^{8}$ However, the DWS has not been used to investigate the properties of corn stover nanocellulose (CSNC).

Because the properties of nanocellulose (NC) highly depend on the source that cellulose comes from, it is necessary to study different NC properties individually. Recently, we developed NC from corn stover agricultural waste. ${ }^{3}$ In addition, we have investigated the mechanical rheological behavior of corn stover nanocellulose (CSNC). ${ }^{9}$ In this work, the microheterogeneity and microrheological properties of the CSNC were investigated using the DWS technique. The conventionally determined dynamic viscoelastic behavior of the CSNC was compared with the results obtained by the DWS approach. We found that the microrheological properties of CSNC measured by DWS were in excellent agreement with the conventional mechanical rheological studies on CSNC. In addition, the study of CSNC by DWS provides not only good complementarity between microrheology and macrorheology, but also some new insight into the physical properties and functions of CSNC. The results from this research will be useful to develop new applications for CSNC.

\section{EXPERIMENTAL}

\section{Material and sample preparation}

CSNC was prepared by the method described in our earlier work. ${ }^{3,9}$ Briefly, corn stover powder was first treated with $4 \mathrm{wt} \%$ sodium hydroxide to remove the soluble polysaccharides. After the alkali treatment, the sample was washed with water and bleached with sodium chlorite and acetic acid. The bleaching process removes most of the lignin or polyphenols and proteins. After bleaching, the product was washed with water and air-dried. This air-dried product contains about $93.1 \%$ cellulose, $2.5 \%$ hemicellulose, and $3.4 \%$ lignin. ${ }^{3,9}$ The dried powder was then suspended in deionized water and subjected to high temperature high pressure homogenization with 30 passes through a GEH NiroSoavi Panda 2K (43100 Parma, Italy), at 500 bars of pressure and a temperature of $90-95^{\circ} \mathrm{C}$. The produced cellulose microfibrils' diameters are in the range of $20-40 \mathrm{~nm}$, indicating that the corn stover cellulose fiber becomes CSNC after the hightemperature high-pressure homogenized shearing. The final CSNC was freeze-dried., ${ }^{3,9}$ The freeze-dried CSNC was resuspended in de-ionized water at a desired concentration for the DWS measurements. Samples were stored at $4{ }^{\circ} \mathrm{C}$ and used within 2 days after preparation to avoid any sample degradation. At least three samples were examined at each CSNC concentration.

\section{Diffusing wave spectroscopy (DWS) measurements}

The diffusing wave spectroscopy (DWS) technology is a modern light scattering technique to characterize turbid media. ${ }^{10} \mathrm{~A}$ laser light beam is focused and incident on a flat scattering cuvette, which contains the polymer material mixed with spherical optical probes. A photon detector collects the multiply scattered light from the sample. The output of the photon detector is directed into a correlator, which will determine the autocorrelation function $\left(g_{2}(t)-1\right)$. From the autocorrelation function $\left(g_{2}(t)-1\right)$, the mean square displacement (MSD) of the probing beads, $\left\langle\Delta \mathrm{r}^{2}(t)\right\rangle$, can be extracted using a root-search algorithm following classical techniques. ${ }^{4}$ In addition, the local rheological behaviors of the polymer solution can be calculated from the MSD via the generalized StokesEinstein equation. ${ }^{7,10}$ Briefly, the local modulus of a viscoelastic polymer solution and the mean square displacement of a microsphere suspended in that fluid are related by:

$$
\tilde{G}(s)=\frac{s}{6 \pi a}\left[\frac{6 \mathrm{k}_{\mathrm{B}} \mathrm{T}}{s^{2}\left(\Delta \tilde{r}^{2}(s)\right)}-m s\right] \approx \frac{\mathrm{k}_{\mathrm{B}} \mathrm{T}}{\pi a s\left(\Delta \tilde{r}^{2}(s)\right)}
$$

where $\mathrm{k}_{\mathrm{B}}$ is Boltzmann's constant, $\mathrm{T}$ is the temperature of the sample, $\alpha$ represents the radius of the microsphere, $m$ is its mass, $s$ represents the Laplace frequency, and $\sim$ is the unilateral Laplace transformation which is defined as:

$$
\tilde{X}(s) \equiv L[X(t)] \equiv \int_{0}^{\infty} X(t) \exp (-s t) d t
$$

Equation (1) relates the unilateral Laplace transformation of the stress relaxation modulus $G_{r}(t), G(s) \equiv s \widetilde{G_{r}}(s)$ to the Laplace transformation of the mean-square displacement $\left\langle\Delta \vec{r}^{2}(t)\right\rangle$. In Equation (1), the inertia term (the second term in the brackets) can be neglected due to its corresponding to very high frequencies. The knowledge of $(s)$ is sufficient to characterize the viscoelastic behavior of a polymer solution. However, in order to present the optical measurements in a more familiar fashion, the analytic 
continuation between the real function $(s)$ and the complex function $G^{*} \equiv G^{i}(\omega)+i G^{\prime \prime}(\omega)$ is used. The storage and loss moduli can be extracted by replacing $\mathrm{s}$ by $\mathrm{i}^{\omega}$ in $\tilde{G}_{(s)}$ and by extracting real and imaginary

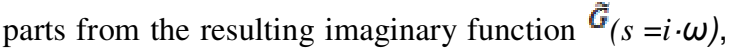
respectively. The diffusion coefficient of the imbedded microspheres can be calculated as $\mathrm{D}(t)=\left\langle\Delta \mathrm{r}^{2}(t)>/ 6 t\right.$. $^{11}$

The DWS measurements on the CSNC were conducted using the DWS RheoLab II instrument (LS Instruments AG, Switzerland). Polystyrene microspheres of $222 \mathrm{~nm}(0.5 \%)$ were gently mixed with the SGRS solution in a cuvette, so that the microspheres were uniformly embedded in the CSNC solution. ${ }^{5,6}$ The temperature of the chamber holding the cuvette in the DWS RheoLab II was controlled at 25 ${ }^{\circ} \mathrm{C} \pm 0.1{ }^{\circ} \mathrm{C}$. The added polystyrene microbeads did not affect the viscoelastic moduli and viscosity of the CSNC solution measured with conventional rheological measurements.

\section{Mechanical rheological measurements}

The method adopted in this work was used many times in our previous work for measurement of biopolymers, such as wheat proteins, ${ }^{12}$ soy proteins, ${ }^{13}$ lupin proteins, ${ }^{14}$ and $\beta$-glucans. ${ }^{15}$ The rheological properties of different concentrations of CSNC were measured by a strain-controlled Rheometric ARES rheometer (TA Instruments, New Castle, DE, USA). The $50-\mathrm{mm}$ diameter plate-plate geometry was adopted. The temperature was controlled at $25 \pm 0.1{ }^{\circ} \mathrm{C}$ by a water circulation system. For linear viscoelastic measurements, to ensure that all the measurements for the materials were made within the linear range for the linear viscoelastic property studies, the strain-sweep experiments were conducted initially. An applied shear strain valued in the linear range was adopted for the other linear viscoelastic property measurements for the same material; fresh samples were used for each experiment. The linear viscoelasticity indicates that the measured parameters are independent of the applied shear strain. Small-amplitude oscillatory shear experiments were conducted over a frequency $(\omega)$ range of $0.1-500 \mathrm{rad} / \mathrm{s}$, yielding the shear storage or elastic $\left(G^{\prime}\right)$ and loss or viscous $(G$ ") moduli. The complex modulus is expressed as $G^{*} \equiv \sigma^{\prime}(\omega)+i G^{\prime}(\omega)$, and the amplitude of the viscoelastic modulus is $\left|G^{*}(\omega)\right|=\left(G^{, 2}+G^{, 2}\right)^{1 / 2}$.

\section{RESULTS AND DISCUSSION}

From the time-dependent mean-square displacements (MSD) of the $222 \mathrm{~nm}$ diameter microbeads embedded in the CSNC solutions detected by DWS, the microrheological properties of the CSNC can be deduced. New insights into the CSNC solution dynamics of relaxation would be obtained from the measurements of the MSDs and associated diffusion coefficients in the temporal domain. The MSDs, $<\Delta \mathrm{r}^{2}(t)>$, were extracted from the measured autocorrelation function $\left(g_{2}(t)-1\right)$ of the light multiple scattered by the microbeads embedded with the CSNC solution. Five concentrations of CSNC solutions, $0.25 \%, 0.5 \%$, $1 \%, 1.5 \%$, and $2 \%$ (wt $\%$ ) were measured. Figure 1 displays the autocorrelation functions $\left(g_{2}(t)-1\right)$ for two concentrations of the CSNC solutions. It can be noticed that the higher concentration CSNC retarded the relaxation of the correlation function intensity. This effect accounts for more CSNC fibril interactions at the greater concentrations.

Figure 2 shows the time-dependent meansquare displacements (MSDs) calculated from the autocorrelation functions of the microbeads embedded in five concentrations of the CSNC solutions. The MSDs of the microspheres embedded in the relatively lower concentration of $0.25 \%$ (wt\%) CSNC solution exhibited nearly a power-law behavior with a slope of about 0.9 (Fig. 2). Accordingly, the diffusion coefficients $(\mathrm{D}(t))$ of the microspheres in the $0.25 \%$ CSNC solution did not change much with increased time scale (Fig. 3). The diffusion coefficients of the microbeads in the $0.25 \% \mathrm{CSNC}$ were $1.63 \mu \mathrm{m}^{2} / \mathrm{s}$ and $1.34 \mathrm{\mu m}^{2} / \mathrm{s}$ at $10^{-4}$ and $10^{-3}$ seconds, respectively (Fig. 3). By comparison, the MSDs for the microbeads embedded in the homogeneous viscous solutions, such as water and glycerol, display a power-law behavior with a slope of one; and the diffusion coefficients of the beads would keep constant over the lag time. ${ }^{8}$ However, the MSDs for the microspheres embedded in the heterogeneity polymer solutions exhibit a time-dependence behavior; and the diffusion coefficients of the microspheres would also depend on the lag time. ${ }^{8,16}$ Using the $\mathrm{D}(t)=$ $\mathrm{k}_{\mathrm{B}} \mathrm{T} / 6 \pi \eta \alpha\left(\mathrm{k}_{\mathrm{B}}\right.$ is the Boltzmann constant, $\mathrm{T}=$ $298 \mathrm{~K}, \eta$ is the viscosity of the solvent, and $\alpha$ is the radius of the microspheres), the same microbeads will have the constant diffusion coefficient of $1.97 \mathrm{\mu m}^{2} / \mathrm{s}$ in water. Thus, the behaviors of the microspheres embedded in the $0.25 \%$ (wt\%) CSNC solution were somewhat close to those in water, but a little different. The diffusion coefficients of the beads were somewhat smaller than those in water and slightly dependent on time. The diffusion coefficients of the microspheres embedded in CSNC solutions were smaller than when embedded in water due to the higher viscosity of the CSNC solutions. Therefore, at relatively 
lower concentrations of $0.25 \%$ (wt $\%$ ), the MSDs for the microbeads embedded in the CSNC solutions were even close to the power law of unity, but slightly deviating from it; and the

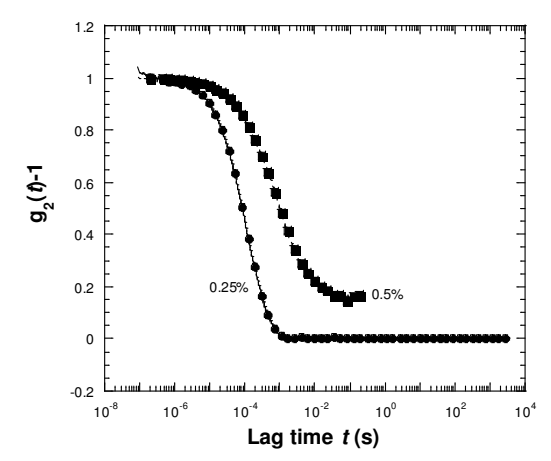

Figure 1: Autocorrelation function of light intensity multiply scattered by $222 \mathrm{~nm}$ diameter microspheres embedded in $0.25 \%(\bigcirc)$ and $0.5 \%$ ( $\square$ ) CSNC solutions at $25^{\circ} \mathrm{C}$

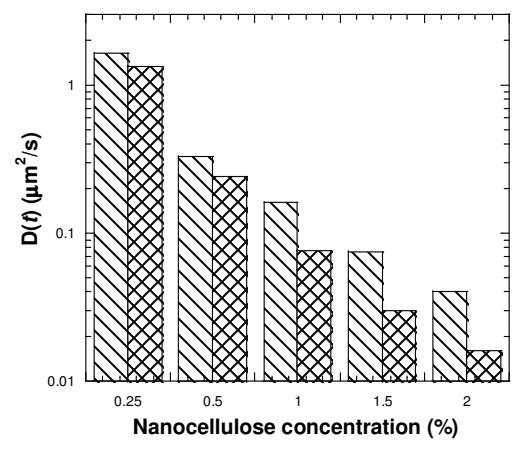

Figure 3: Diffusion coefficient, $\mathrm{D}(t)=\left\langle\Delta \mathrm{r}^{2}(t)>/ 6 t\right.$, of microspheres embedded in five measured concentrations of CSNC solutions at lag time of $10^{-}$ ${ }^{4}$ and $10^{-3}$ seconds and temperature of $25^{\circ} \mathrm{C}$ (the first column: $10^{-4} \mathrm{~s}$; the second column: $10^{-3} \mathrm{~s}$ )

For the $0.5 \%$ (wt\%) CSNC solutions, the MSD of the microspheres embedded in the sample displayed a slope that deviated more from unity (Fig. 2). The diffusion coefficients of the microspheres embedded in the $0.5 \%$ (wt $\%$ ) CSNC were $0.33 \mu \mathrm{m}^{2} / \mathrm{s}$ and $0.24 \mu \mathrm{m}^{2} / \mathrm{s}$ at $10^{-4}$ and $10^{-3}$ seconds, respectively (Fig. 3), which were more dependent on time, implying that the $0.5 \%$ CSNC solution showed more heterogeneity. ${ }^{5,8}$ Apparently, more chain-chain interactions or overlapping occurred between $0.25 \%$ (wt $\%$ ) and $0.5 \%$ (wt $\%$ ). At relatively higher concentrations of CSNC solutions, of diffusion coefficients were a bit time-dependent. Thus, the CSNC solutions exhibited slightly heterogeneity behavior, even at relatively low concentration of $0.25 \%$.

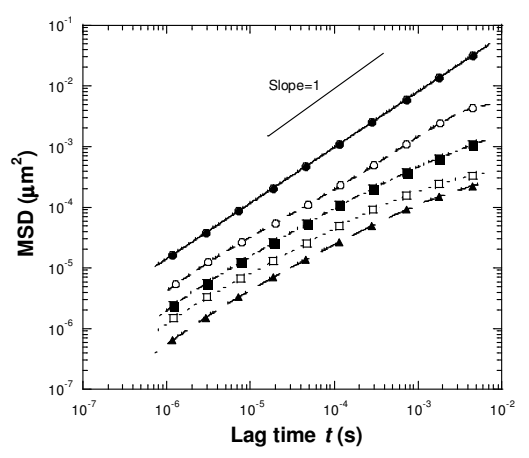

Figure 2: Mean square displacement (MSD), $<\Delta \mathrm{r}^{2}(t)>$, of $222 \mathrm{~nm}$ microspheres embedded in $0.25 \%$ $(\mathbf{O}), 0.5 \%(\bigcirc), 1 \%(\square), 1.5 \%(\square)$, and $2 \%(\mathbf{\Delta})$ CSNC solutions at $25{ }^{\circ} \mathrm{C}$; the straight line shows a slope of one

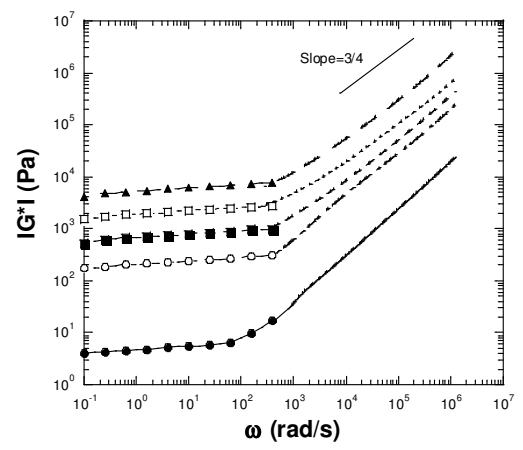

Figure 4: Magnitude of frequency dependence viscoelastic moduli $\left(\left|G^{*}(\omega)\right|\right)$ of $0.25 \%(\bigcirc), 0.5 \%(\bigcirc)$, $1 \%(\square), 1.5 \%(\square)$, and $2 \%(\boldsymbol{\Delta})$ of CSNC solutions measured by both mechanical rheometer and DWS at $25^{\circ} \mathrm{C}$ (symbols are experimental data measured by the mechanical rheometer; lines are data measured by DWS; straight lines show a slope of 3/4

$1 \%, 1.5 \%$, and $2 \%$ (wt $\%$ ), the MSDs of the microspheres mixed with samples further deviated with the unity slope of the power law (Fig. 2). Hence, the diffusion coefficients of the beads were much more dependent on time. For instance, the diffusion coefficient of the beads in the $1.5 \%$ (wt\%) CSNC was 0.075 $\mu \mathrm{m}^{2} / \mathrm{s}$ at $10^{-4}$ second, but rapidly decreased to $0.030 \mu \mathrm{m}^{2} / \mathrm{s}$ at $10^{-3}$ second. The diffusion coefficient of the microspheres embedded in the $2 \%$ (wt $\%$ ) CSNC was $0.040 \mu \mathrm{m}^{2} / \mathrm{s}$ at $10^{-4}$ second, but sharply dropped to $0.016 \mu \mathrm{m}^{2} / \mathrm{s}$ at $10^{-3}$ second (Fig. 3). These results are in 
agreement with the fact that greater concentration CSNC solution exhibited higher viscosity. The extent of the displacements of the microspheres in the CSNC solutions was largely reduced with increasing concentration. The above results of the diffusion coefficient of microspheres dispersed in high concentrations CSNC solutions decreasing with time scale also indicated that the CSNC structure depended on the distance scale. Apparently, with the increasing concentration, the CSNC solution had a transition from slight heterogeneity to more pronounced heterogeneity behavior.

Figure 4 shows the five concentrations of CSNC solutions' amplitude of moduli $\left(\left|G^{*}(\omega)\right|\right)$ over the frequency measured by DWS and mechanical rheometer. The overlapped data indicated that excellent agreement was observed for the measurements between DWS and conventional mechanical rheometer (Fig. 4). These results also implied that DWS measurements can accurately detect high-frequency range viscoelastic properties of CSNC solutions. The amplitude of the viscoelastic moduli $\left(\left|G^{*}(\omega)\right|\right)$ for the CSNC increased with the increasing concentration the higher the concentration, the stronger the CSNC viscoelastic properties (Fig. 4). Apparently, a network of CSNC was established even at a lower concentration, as $0.25 \%$. The CSNC chain-chain interactions became stronger and stronger with the increasing concentration, as evidenced by the greater amplitude of the viscoelastic moduli $\left(\left|G^{*}(\omega)\right|\right)$. We also noticed the high-frequency moduli behaviors of CSNC measured by DWS (Fig. 4). The high-frequency range viscoelastic properties show the early dynamics of relaxation of the CSNC networks. The optical measurements by DWS showed that the amplitude of the viscoelastic moduli $\left(\left|G^{*}(\omega)\right|\right)$ for all measured concentrations of CSNC samples were proportional to 0.75 power of the frequency $\left(\left|G^{*}(\omega)\right| \propto \omega^{0.75}\right)$. Conventional mechanical rheology measurements of the frequency dependence of the moduli for $\omega>$ $300 \mathrm{rad} / \mathrm{s}$ are extremely limited, therefore direct comparison with published data is difficult at high frequencies. Moreover, our strain-controlled rheometer can measure the moduli only up to about $300 \mathrm{rad} / \mathrm{s}$, so a direct comparison of our DWS measurements with our mechanical measurements at high frequency is also precluded. Some theoretical models predicted the high-frequency dependence of $\left|G^{*}(\omega)\right|$. In addition to the fact that the measurement of the frequency dependence of $\left|G^{*}(\omega)\right|$ provides us with a much more rigorous test of current dynamical models of CSNC dynamics. The highfrequency regime also offers a new insight into the local dynamics of CSNC fibril inside their confinement tube.

Morse $^{17-19}$ has established a model, which specifically describes the rheological behavior and dynamics of semiflexible polymers in solution. This model predicts that at large frequencies, $\left|G^{*}(\omega)\right| \propto \omega^{\alpha}$ with $\alpha=0.75$, which is in excellent consistency with our DWS measurements for CSNC. This exponent $\alpha$ is much larger than the one describing the largefrequency behavior of flexible polymer, for which $\alpha=0.5 .^{20}$ The molecular interpretation of rheological properties is not as well understood as other modern biochemical and molecular biology techniques, because the molecular interpretation of rheological properties is relatively 'indirect', while molecular interpretation using biochemical and molecular biology techniques is relatively 'direct'. Our above DWS studies on CSNC indicated that CSNC exhibited semi-flexible behavior. There are very few reports about the physical properties of CSNC. ${ }^{9}$ The technique of DWS has allowed us to monitor the very high-frequency viscoelastic moduli of CSNC in a single experiment at a fixed temperature, without disturbing the sample, which cannot be done by using the mechanical rheometer. From these high-frequency measurements, a new insight into the mechanism of early dynamics of stress relaxation in entangled networks and an understanding of the type of local macromolecular motion for $\mathrm{CSNC}$ can be obtained.

\section{CONCLUSION}

In this work, we studied the microheterogeneity microrheological properties of CSNC solutions using the DWS technique and compared the rheological properties measured by both DWS and conventional mechanical rheometry. We observed the heterogeneity of CSNC increased with the increasing concentration. At low 
concentration of $0.25 \%$, the CSNC solutions exhibited some heterogeneity. The heterogeneity became stronger when the CSNC concentration was higher, because the diffusion coefficients $(\mathrm{D}(t))$ of the microspheres embedded in CSNC were more strongly dependent on time. In addition, we found that the rheological data measured by both DWS and mechanical rheometry exhibited excellent agreement with each other. CSNC exhibited concentration-dependent rheological properties - the higher the concentration, the stronger the viscoelastic behavior. The high-frequency magnitude of the viscoelastic moduli $\left(\left|G^{*}(\omega)\right|\right)$ for the CSNC solutions studied by DWS can be characterized as $\left|G^{*}(\omega)\right| \propto \omega^{0.75}$, which entails a semiflexible polymer behavior.

ACKNOWLEDGMENT: This work was financially supported by the US Department of Agriculture, Agricultural Research Service.

Mention of trade names or commercial products in this publication is solely for the purpose of providing specific information and does not imply recommendation or endorsement by the U.S. Department of Agriculture. USDA is an equal opportunity provider and employer.

\section{REFERENCES}

1 M. Rajinipriya, M. Nagalakshmaiah, M. Robert and S. Elkoun, ACS Sustain. Chem. Eng., 6, 2807 (2018), https://doi.org/10.1021/acssuschemeng.7b03437

2 H. Chen, "Biotechnology of Lignocellulose", Springer, 2014, pp. 25-71

J. Xu, E. F. Krietemeyer, V. M. Boddua, S. X. Liu and W.-C. Liu, Carbohyd. Polym., 192, 202 (2018), https://doi.org/10.1016/j.carbpol.2018.03.017

4 D. Weitz and D. J. Pine, in "Dynamic Light Scattering: the Method and Some Applications", edited by W. Brown, Oxford University Press, Oxford England, New York, 1993

5 J. Xu, G. E. Inglett, S. X. Liu and V. M. Boddu, Food Biophys., 11, $339 \quad$ (2016), https://doi.org/10.1007/s11483-016-9447-2

6 J. Xu, A. Palmer and D. Wirtz, Macromolecules, 31, $6486 \quad$ (1998), https://doi.org/10.1021/ma9717754

7 T. G. Mason, Rheol. Acta, 39, 371 (2000), https://doi.org/10.1007/s003970000094
8 J. Xu, Y. Tseng, C. J. Carriere and D. Wirtz, Biomacromolecules, $\quad 3, \quad 92 \quad$ (2002), https://doi.org/10.1021/bm015586b

9 J. Xu, W.-C. Liu and V. M. Boddu, Cellulose Chem. Technol., 52, $337 \quad$ (2018), http://www.cellulosechemtechnol.ro/pdf/CCT56(2018)/p.\%20337-342.pdf

10 T. G. Mason and D. A. Weitz, Phys. Rev. Lett., 74, 1250 (1995), https://doi.org/10.1103/PhysRevLett.74.1250

11 J. Xu, V. Viasnoff and D. Wirtz, Rheol. Acta, 37, $387 \quad$ (1998), https://doi.org/10.1007/s003970050125

12 J. Xu, J. A. Bietz, F. C. Felker, C. J. Carriere and D. Wirtz, Cereal Chem., 78, 181 (2001), https://doi.org/10.1094/CCHEM.2001.78.2.181

13 J. Xu, A. A. Mohamed, M. P. HojillaEvangelista and D. J. Sessa, Am. J. Food Tech., 3, 294 (2008), https://scialert.net/abstract/?doi=ajft.2008.294.302

14 J. Xu, A. A. Mohamed and D. J. Sessa, Int. J. Agric. Res., 3, $317 \quad$ (2008), https://scialert.net/abstract/?doi=ijar.2008.317.324

15 J. Xu, G. E. Inglett, D. Chen and S. X. Liu, Food Chem., 138, $186 \quad$ (2013), https://doi.org/10.1016/j.foodchem.2012.10.049

16 A. Palmer, J. Xu and D. Wirtz, Rheol. Acta, 37, 97 (1998), https://doi.org/10.1007/s003970050095

17 D. C. Morse, Phys. Rev. E, 58, R1237 (1998), https://doi.org/10.1103/PhysRevE.58.R1237

18 D. C. Morse, Macromolecules, 31, 7030 (1998), https://doi.org/10.1021/ma9803032

19 D. C. Morse, Macromolecules, 31, 7044 (1998), https://doi.org/10.1021/ma980304u

${ }^{20}$ M. Doi and S. F. Edwards, in "The Theory of Polymer Dynamics", Oxford University Press, London, 1986 